\title{
Carbohydrate and lactate utilisation during exercise with and without Yerba Maté ingestion
}

Nutritional ergogenic and thermogenic aids are commonly used to optimise carbohydrate utilisation and potentially enhance glycogen-sparing ability ${ }^{(1-2)}$. However, less is known about the potential effects during exercise, particularly on the responses of carbohydrate oxidation (CHO) and blood lactate concentration (BLC), which are known indicators of exercise intensity and determine exercise performance ${ }^{(3)}$. This study investigated whether the potential thermogenic effects of Yerba Maté (YM), the plant of (Illex Paraguariensis), affect carbohydrate oxidation rate (CHO) and BLC levels during low and moderate intensity exercise.

Male and female participants $(n=11)$ ingested either $1 \mathrm{~g}$ of YM or placebo capsules (PLA) in a randomised crossover experimental design. Within laboratory conditions, participants rested for $1 \mathrm{hr}$ before performing two incremental exercise ergometry tests in separate visits with the power output being initiated and increased by $0.5 \mathrm{~W} \cdot \mathrm{kg}^{-1}$ of body mass every 3 min stage until exhaustion. Cardiorespiratory measurements and indirect calorimetry technique were applied to analyse CHO and energy expenditure derived from carbohydrate $\left(\mathrm{EE}_{\mathrm{cho}}\right)$, and capillary blood samples were collected and analysed for BLC at rest and for each exercise intensity domain. Data were analysed using a repeated measures ANOVA design.

$\mathrm{CHO}$ was reduced significantly (e.g. $0 \cdot 37 \pm 0.24$ vs. $0.59 \pm 0 \cdot 19,0 \cdot 75 \pm 0.24$ vs. $1.00 \pm 0 \cdot 33$ and $1.36 \pm 0.48$ vs. $1.64 \pm 0.47$ g.min ${ }^{-1}$ at 40, 50, 60 and $70 \%$ of $\dot{V} O_{2}$ peak respectively, $P<0 \cdot 001$, ANOVA main effects) in the YM compared with PLA at all sub-maximal exercise intensities up to the level of $70 \%$ of maximal oxygen uptake $\left(\dot{V} O_{2 \text { peak }}\right)$, which corresponded to reaching carbohydrate saturation level (respiratory exchange ratio $=1),\left(p<0.001\right.$, ANOVA main effects). $\mathrm{EE}_{\mathrm{CHO}}$ was also reduced in the YM compared with PLA (Table1). These effects were combined by a trend, though not significant towards a decrease in BLC $(P=0 \cdot 07)$ at the same given exercise intensities in YM compared with PLA (Table 1).

Table 1. Carbohydrate oxidation rate $(\mathrm{CHO})$ and Energy derived from carbohydrate $\left(\mathrm{EE}_{\mathrm{CHO}}\right)$ at low to moderate exercise intensities, all data presented as (Mean $\pm \mathrm{SD})$.

\begin{tabular}{|c|c|c|c|c|}
\hline $\begin{array}{l}\text { Intensity } \\
\left.\text { (Relative to } \dot{V} O_{2 \text { peak }} \%\right)\end{array}$ & $\begin{array}{l}\mathrm{EE}_{\mathrm{CHO}-\mathrm{YM}} \\
\left(\mathrm{kcal}_{\mathrm{min}}^{-1}\right)\end{array}$ & $\begin{array}{l}\mathrm{EE}_{\mathrm{CHO}-\mathrm{PLC}} \\
\left(\mathrm{kcal}_{\mathrm{min}}^{-1}\right)\end{array}$ & $\begin{array}{l}\text { BLC-YM } \\
\text { mmol.1 }\end{array}$ & $\begin{array}{l}\text { BLC-PLC } \\
\text { mmol. } 1^{-1}\end{array}$ \\
\hline 40 & $1.47 \pm 1.00$ & $2.37 \pm 0.78$ & $1 \cdot 7 \pm 0 \cdot 5$ & $1.7 \pm 0.4$ \\
\hline 50 & $3.01 \pm 0.97$ & $4 \cdot 00 \pm 1 \cdot 33$ & $2 \cdot 5 \pm 0.7$ & $2.4 \pm 0.7$ \\
\hline 60 & $5.40 \pm 1.91$ & $6 \cdot 58 \pm 1.90$ & $2 \cdot 7 \pm 1 \cdot 2$ & $3 \cdot 0 \pm 1 \cdot 3$ \\
\hline 70 & $8.87 \pm 3.22$ & $10 \cdot 06 \pm 2 \cdot 23$ & $3 \cdot 5 \pm 2 \cdot 0$ & $4 \cdot 1 \pm 2 \cdot 5$ \\
\hline
\end{tabular}

Reduced $\mathrm{CHO}, \mathrm{EE}_{\mathrm{cho}}$ seem to be reflected by BLC changes at moderate exercise intensities, through acute $\mathrm{YM}$ ingestion at submaximal exercise intensities. These effects suggest a potential role for YM during exercise for glycogen sparing, exercise tolerance and metabolic weight loss outcomes.

1. Cermak NM, van Loon LJ (2013) Sports Med 43(11), 139-55.

2. Outlaw J, Wilborn C, Smith A et al. (2013) J Int Soc Sports Nutr 10(1), 25.

3. Alkhatib A (2010) Lactate, carbohydrate, and fat utilisation during exercise, LAP, Germany. 